\title{
$\left.{ }^{10} \mathrm{~B}(n, \alpha)\right)^{7} \mathrm{Li}$ 和 ${ }^{6} \mathrm{Li}(n, \alpha){ }^{3} \mathrm{H}$ 反应对青藏高原 某些盐湖中硼和锂同位素组成的影响
}

\author{
肖应凯 \\ （中国科学院青海盐湖研究所，西宁）
}

\section{一、前 立}

天然来源的硼和锂同位素组成已有不少报道. McMullen ${ }^{[1]}$ 测定了美国西尔兹湖样品中的 绝对 ${ }^{11} \mathrm{~B} /{ }^{10} \mathrm{~B}$ 比值为 $4.040-4.072$, 他指出以前所发表的偏高的数值可能是测量误差引起的. Schwarcz ${ }^{[2]}$ 等指出陆地样品的 ${ }^{11} \mathrm{~B} /{ }^{10} \mathrm{~B}$ 比值在 4.0 左右, 而海水的 ${ }^{11} \mathrm{~B} /{ }^{10} \mathrm{~B}$ 比值相差很远 (4.2), 此 络论与 Shima ${ }^{[3]}$ 的不一致. Shima 发现陆地矿石和海水的 ${ }^{11} \mathrm{~B} /{ }^{10} \mathrm{~B}$ 比值大约为 $4.05 \pm 0.05$, 而大 气样品的 ${ }^{11} \mathrm{~B} /{ }^{10} \mathrm{~B}$ 比值大约要低 3-6\%. Harry ${ }^{[3]}$ 等列出了发表过的 ${ }^{7} \mathrm{Li} /{ }^{6} \mathrm{Li}$ 比值, 他认为 ${ }^{7} \mathrm{Li} /{ }^{6} \mathrm{Li}$ 比值大的变化可能是仪器因素和天然变化的结果, Harry 等测定了来自北美和非洲的天然样 品中锂同位紊的绝对比值, 30 个锂辉石样品的平均 ${ }^{7} \mathrm{Lj} /{ }^{6} \mathrm{Lj}$ 比值是 $12.22, \mathrm{Li}_{2} \mathrm{CO}_{3}$ 试剂的为 12.62 , 而美国西尔兹湖中的 ${ }^{7} \mathrm{Li} /{ }^{6} \mathrm{Li}$ 比值是 12.57 .

在宇宙射线中热中子作用下会发生两种核反应: $\left.{ }^{10} \mathrm{~B}(n, \alpha)\right)^{7} \mathrm{Li}$ 和 ${ }^{6} \mathrm{Li}(n, \alpha)^{3} \mathrm{H}$ ，这两个反 应使得 ${ }^{10} \mathrm{~B}$ 丰度降低和 ${ }^{7} \mathrm{Li}$ 丰度增高, 其结果必然会影响硼和锂同位素的组成. 但是直到目前 还没有人从实验上证实这一点.

由于我国青藏高原独特的地理条件，为研究研和锂同位素组成受宇宙射线的作用而产生 的变化提供了充分的可能性. 本文在这方面进行了初步研究.

\section{二、实验}

方交换法分或国为了从盐湖水中分离嗍，将强酸性阳离子交换树脂(氢型)和弱碱 性阴离子交换树脂“离子交换剂 11”（碳酸氢根型)装在一特殊的交换柱中,盐湖水样品用盐酸 酸化后,注人到交换柱中,立即振荡 10 分钟, 被吸附的砋用 60 毫升纯水洗涤. 砞的回收大于 $98 \%$ ，硼中总的杂质含量小于 $2 \%$. 所有的试剂必须是无嗍的, 所有器血均采用石英或聚乙 烯制品．在制样时矹同位素分馏可忽略. 样品中的矹含量用甘露醇碱滴定法测定.

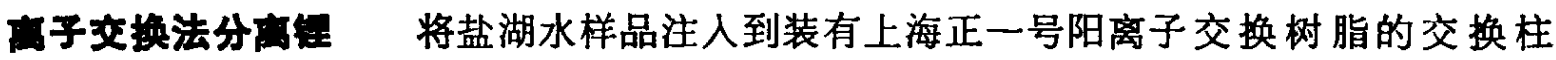
中，被柱中树脂所吸附的锂用 $1.2 \mathrm{M} \mathrm{HCl}-80 \%$ 甲醇混合溶液淋洗，150-350微克锂的回收大 于 $97 \%$ ，可分离的镁量为 200 毫克，而钠量可不受限制，此过程中的锂同位素分馏可忽略 （包头 202 厂提供的 $\mathrm{Li}_{2} \mathrm{CO}_{3}$ 样品经以上处理前后测得的 ${ }^{6} \mathrm{Li}$ 丰度值分别为 $7.58 \pm 0.02 \%$ 和 
$7.58 \pm 0.01 \%)$. 样品中锂的含量用原子吸收分光光度法测定.

同位意分析 ${ }^{11} \mathrm{~B} /{ }^{10} \mathrm{~B}$ 比值采用喼砂的表面电离方法测定, 所用仪器是经改进后的 $2 \mathrm{hT}-$ 1301 型质谱计 ${ }^{[5,61}$, 没有观察到有分馏和记忆效应, 测量精度为 $\pm 0.3 \%$, 测定结果列于表 1 .

表 1 青藏高原某些盐湖中的 ${ }^{11} \mathrm{~B} /{ }^{10} \mathrm{~B}$ 比值

\begin{tabular}{|c|c|c|c|}
\hline 样 品 & 测定次数 & 平均 ${ }^{11} \mathrm{~B} /{ }^{10} \mathrm{~B}$ 值 & 样 品 产 地 \\
\hline 1 & 2 & 4.107 & 青海一里坪盐湖水 \\
\hline 2 & 3 & 4.086 & 音海台吉乃尔盐湖水 \\
\hline 3 & 4 & 4.097 & 青海大策旦盐湖水 \\
\hline 4 & 3 & 4.068 & 青海大柴旦温泉水 \\
\hline 5 & 7 & 4.071 & 西藏张藏水卡 I 盐湖水 \\
\hline 6 & 4 & 4.081 & 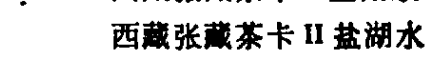 \\
\hline 7 & 4 & 4.068 & 西荿斑戈搵湖水 \\
\hline 8 & 3 & 4.073 & 西㵧扎湖盐湖水 \\
\hline
\end{tabular}

测定 ${ }^{7} \mathrm{Lj} /{ }^{6} \mathrm{Li}$ 比值时, 首先将分离出来的锂转化成 $\mathrm{LiNO}_{3},{ }^{7} \mathrm{Li} /{ }^{6} \mathrm{Li}$ 比值用经过改进后的 ZhT-1301 型质谱计测定. $\mathrm{LiNO}_{3}$ 涂在离子源中的两侧带上, 它被来自中央带的辐射热加热并 蒸发, 随后在中央带上电离成锂离子, 再经加速, 磁场分离, 最后收集在法拉第接收器上, 采用 磁场跳扫扫描谱峰. 放大后的锂离子流用数字电压表测量. 测量中没有发现分馏和记々效 应, 测量精度为 $\pm 0.2 \%$ ，结果列于表 2 .

表 2 青藏高原某些盐湖中 ' $\mathrm{Li} /{ }^{\circ} \mathrm{Li}$ 比值

\begin{tabular}{|c|c|c|c|}
\hline 样 品 & 测定次数 & 平均 ${ }^{\prime} \mathrm{Li} /{ }^{\circ} \mathrm{Li}$ 值 & 样 品 产 地 \\
\hline 1 & 6 & $12.29 \pm 0.021$ & 青海一里坪盐湖水 \\
\hline 2 & 5 & $12.35 \pm 0.005$ & 青海台吉乃尔搵湖水 \\
\hline 3 & 5 & $12.42 \pm 0.016$ & 海大柴旦盐湖水 \\
\hline 4 & 5 & $12.22 \pm 0.019$ & 青海大恝旦温泉水 \\
\hline 5 & 5 & $12.32 \pm 0.029$ & 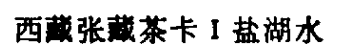 \\
\hline 6 & 5 & $12.36 \pm 0.019$ & 西藏张藏茶卡 II 盐湖水 \\
\hline 7 & 5 & $12.39 \pm 0.017$ & 西荿班戈盐湖水 \\
\hline
\end{tabular}

\section{三、讨 论}

某些盐湖水样品的 ${ }^{11} \mathrm{~B} /{ }^{10} \mathrm{~B}$ 和 ${ }^{7} \mathrm{Li} /{ }^{6} \mathrm{Li}$ 比值都列于表 3, 样品中的硼和锂的含量以及它们的 比值也列于表 3, 这些结果并表示在图 1 中.

1. 表 3 和图 1 指出, 青海高原盐湖的 ${ }^{11} \mathrm{~B} /{ }^{10} \mathrm{~B}$ 比值要比西藏高原盐湖的 ${ }^{11} \mathrm{~B} /{ }^{10} \mathrm{~B}$ 比值高, 这与 它们的沉积年代有关. 在引起地表盐湖硼同位害变化的因素中, ${ }^{10} \mathrm{~B}(n, \alpha)^{7} \mathrm{Li}$ 反应是其中的重 要因来. 我们知道, 宇宙射线中的中子到达地面时部分已减速成慢中子, 另外一些快中子在穿 过水层时也会减速成慢中子, 盐湖水中的 ${ }^{10} \mathrm{~B}$ 在慢中子的作用下会发生 ${ }^{10} \mathrm{~B}(n, \alpha){ }^{7} \mathrm{Li}$ 反应, 引起 ${ }^{10} \mathrm{~B}$ 丰度的减少. 十分明显, 经受 ${ }^{10} \mathrm{~B}(n, \alpha){ }^{7} \mathrm{Li}$ 反应的时间越长, ${ }^{11} \mathrm{~B} /{ }^{10} \mathrm{~B}$ 比值的变化就会更加显 著. 青藏高原盐湖地质年代研究表明 ${ }^{[7]}$, 青海柴达木盆地盐湖开始成盐期为更新世, 而西藏盐 湖比较年轻, 直到全新世才出现明显地沉积, 与此同时盐湖水中的硼才开始集中, 只有当硼集 中到盐湖中以后才会有更多的机会受到宇宙射线中中子的照射, ${ }^{11} \mathrm{~B} /{ }^{10} \mathrm{~B}$ 比值才开始变化. 十 分明显, 由于柴达木盆地盐湖形成时期要比西藏盐湖的早, 因此前者受 ${ }^{10} \mathrm{~B}(n, \alpha){ }^{7} \mathrm{Li}$ 反应影响 
表 3 某些盐湖样品中的 ${ }^{11} \mathrm{~B} /{ }^{10} \mathrm{~B} 、{ }^{\prime} \mathrm{Li} /{ }^{\circ} \mathrm{Li}$ 比值和硼锂的含量

\begin{tabular}{|c|c|c|c|c|c|c|c|}
\hline 样品 & 产 地* & 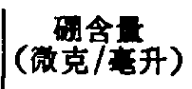 & (锤克含哯升) & $\begin{array}{l}\text { 砶/锂 } \\
(\mathbf{S})\end{array}$ & 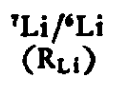 & $\begin{array}{c}{ }^{11} \mathrm{~B} /{ }^{10} \mathrm{~B} \\
\left(\mathrm{R}_{\mathrm{B}}\right)\end{array}$ & $\begin{array}{l}R_{L_{L} / R_{B}}(R) \\
(R)\end{array}$ \\
\hline 1 & 青海高原 & 528 & 293.8 & 1.80 & 12.29 & 4.107 & 3.00 \\
\hline 2 & 海高原 & 998 & 366.3 & 2.72 & 12.35 & 4.086 & 3.02 \\
\hline 3 & 霄海高原 & 1441 & 114.4 & 12.60 & 12.42 & 4.097 & 3.03 \\
\hline 4 & 青海高原 & 40 & 3.5 & 11.43 & 12.22 & 4.068 & 3.00 \\
\hline 5 & 西酢高原 & 934 & 781.3 & 1.20 & 12.32 & 4.071 & 3.03 \\
\hline 6 & 西政高原 & 949 & 409.4 & 2.32 & 12.36 & 4.081 & 3.03 \\
\hline 7 & 西娍高原 & 897 & 112.5 & 7.97 & 12.39 & 4.068 & 3.04 \\
\hline
\end{tabular}

*样品号与产地均与衰 $1 、$ 表 2 相同.

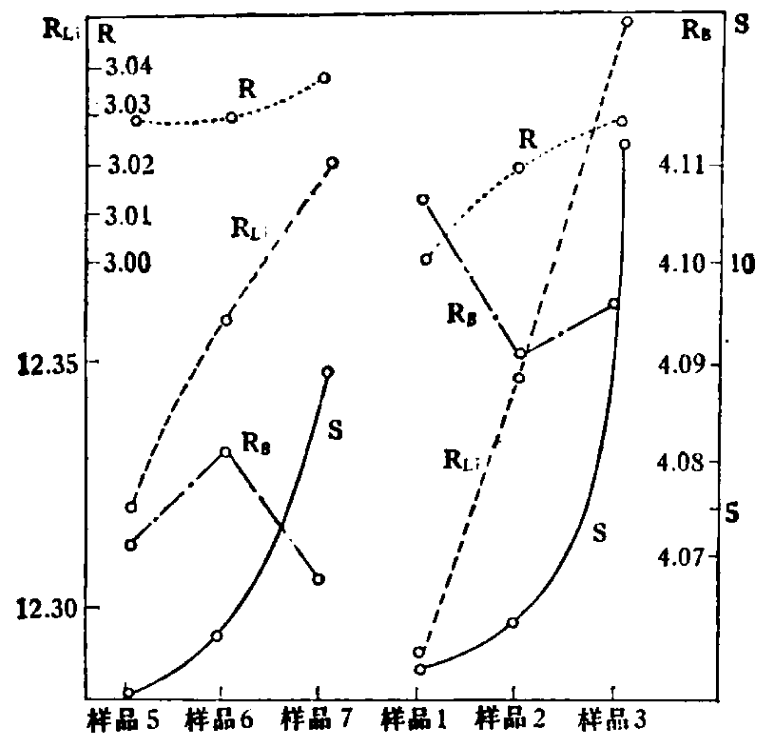

图 1 青海和西藏盐湖中 ${ }^{11} \mathrm{~B} /{ }^{10} \mathrm{~B} 、{ }^{7} \mathrm{Li} /{ }^{\circ} \mathrm{Li}$ 和硼锂含量的关系

的积累时间要长, 由此而造成的 ${ }^{10} \mathrm{~B}$ 丰度的相对减少就会更加明显.

2. 宇宙射线中中子的穿透能力是有限的, 在地球深层, 中子的辐射强度很弱, 很少有 ${ }^{10} \mathrm{~B}(n$, $\alpha)^{7} \mathrm{Li}$ 反应发生(除非有其他的中子源), 因此在地球的深层, ${ }^{11} \mathrm{~B} /{ }^{10} \mathrm{~B}$ 和 ${ }^{7} \mathrm{Li} /{ }^{6} \mathrm{Li}$ 比值不会受到上 述反应的影响. 大柴旦温泉水来自地球的深层, 此处的 ${ }^{11} \mathrm{~B} /{ }^{10} \mathrm{~B}$ 和 ${ }^{7} \mathrm{Li} /{ }^{6} \mathrm{Li}$ 比值应该低于受中子 较强照射的大柴旦湖的 ${ }^{11} \mathrm{~B} /{ }^{10} \mathrm{~B}$ 和 ${ }^{7} \mathrm{Li} /{ }^{6} \mathrm{Li}$ 比值, 测定结果证实了这一点, 大柴旦温泉水的 ${ }^{11} \mathrm{~B} /$ ${ }^{10} \mathrm{~B}$ 和 ${ }^{7} \mathrm{Li} /{ }^{6} \mathrm{Li}$ 比值都要明显地低于相邻近的大柴目湖的 ${ }^{11} \mathrm{~B} /{ }^{10} \mathrm{~B}$ 和 ${ }^{7} \mathrm{Li} /{ }^{6} \mathrm{Li}$ 比值. 如果将此归结 于温泉水在地球深层迁移中的同位素分馏,理由可能是不充分的,因为使 ${ }^{10} \mathrm{~B}$ 和 ${ }^{6} \mathrm{Li}$ 同时富集的 因亲不大可能同时存在.

3. 锂同位亲比值的变化远比嗍同位素比值的变化复杂, 这是因为它同时受到 ${ }^{10} \mathrm{~B}(n, \alpha)^{7} \mathrm{Li}$ 和 ${ }^{6} \mathrm{Li}(n, \alpha)^{3} \mathrm{H}$ 两个反应的影响. 它主要受到 $\mathrm{B} / \mathrm{Li}$ 比值的限制. 在一般盐湖水中, $\mathrm{B} / \mathrm{Li}$ 比值 都要大于 1 , 而且 ${ }^{10} \mathrm{~B}(n, \alpha)^{7} \mathrm{Li}$ 反应的热中子吸收截面是 3837 蚆, 远比 ${ }^{6} \mathrm{Li}(n, \alpha)^{3} \mathrm{H}$ 反应的大 (940 蚆) ${ }^{[0]}$, 因此 ${ }^{10} \mathrm{~B}(n, \alpha)^{7} \mathrm{Li}$ 反应的影响比 ${ }^{6} \mathrm{Li}(n, \alpha)^{3} \mathrm{H}$ 反应的影响要大得多. 从图 1 可见,在 相同的地质年龄条件下, ${ }^{7} \mathrm{Li} /{ }^{6} \mathrm{Li}$ 比值随矹/锂比值的增加而逐渐增加, 这时, ${ }^{7} \mathrm{Li}$ 相对丰度的增 加主要是来自 $\left.{ }^{10} \mathrm{~B}(n, \alpha)\right)^{7} \mathrm{Li}$ 反应的贡献, 硼/锂比值越高, ${ }^{10} \mathrm{~B}(n, \alpha){ }^{7} \mathrm{Li}$ 反应的贡献越大, 所以 ${ }^{7} \mathrm{Li} /{ }^{6} \mathrm{Li}$ 比值越高. 


\section{四、结语}

我国青藏高原海拔高，宇宙射线强，分布着各种类型的地表盐湖，而它们的沉积年代又不 相同, 这就为研究我国青藏高原盐湖中硼和锂同位素组成受 ${ }^{10} \mathrm{~B}(n, \alpha){ }^{7} \mathrm{Li}$ 和 ${ }^{6} \mathrm{Li}(n, \alpha){ }^{3} \mathrm{H}$ 反应 的影晌提供了充分的可能性. 结果表明, 柴达木盆地的盐湖的 ${ }^{11} \mathrm{~B} /{ }^{10} \mathrm{~B}$ 比值比西藏盐湖的高; 在 相同地质年龄条件下, ${ }^{7} \mathrm{Li} /{ }^{6} \mathrm{Li}$ 比值主要由矹/锂比值决定, 且随硼/锂比值的增高而增高. 青 藏高原盐湖中硼锂同位素组成的这种变化, 可能是在宇宙射线作用下发生的 ${ }^{10} \mathrm{~B}(n, \alpha){ }^{7} \mathrm{Li}$ 和 ${ }^{6} \mathrm{Li}(n, \alpha){ }^{3} \mathrm{H}$ 反应引起的.

致谢: 王䓚慧、曹海震、王瑞陵等参加了同位素和元素的测定工作;张彭素对本研究进行了指导，并对本 文提出过修改意见;本所一室提供了盐湖水样品,作者在此一并表示谢意。

\section{\% 文 䘞}

[1] MeMullen, C. C. et al., Geochim et Cosmochim Acta, 23(1961), 147.

[2] Schwarcz, H. P. et al., Earth Planet. Sci. Letters, 6(1969), 1.

[ 3 ] Makoto Shima, Geochim. et Cosmochim. Acta, 27(1963), 911.

[4] Harry, J. Svec et al., Geochim. Cosmochim. Acta, 29(1965), 633.

[5] 肖应凯等, 质徣, 1981, 1: 76 .

[6] 肖应凯, 分析仪器, 1981, 2: 78.

[7] Huang Qi et al., Kexue Tongbao, 26(1981). 1: 66.

[ 8] 椟采图表编制组，核素常用数据表，原子能出版社，1977，3-4. 